D) Check for updates

Cite this: Dalton Trans., 2021, 50 1672

Received 7th December 2020, Accepted 24th December 2020 DOI: $10.1039 / \mathrm{d} 0 \mathrm{dt} 04158 \mathrm{k}$

rsc.li/dalton

\section{ZnAIMCM-41: a very ecofriendly and reusable solid acid catalyst for the highly selective synthesis of 1,3-dioxanes by the Prins cyclization of olefins $\uparrow$}

\author{
Manickam Selvaraj, (D) *a Mohammed A. Assiri, ${ }^{a}$ Hari Singh, ${ }^{b}$ \\ Jimmy Nelson Appaturi, (DD ${ }^{c}$ Ch. Subrahmanyam (D) ${ }^{d}$ and Chang-Sik Ha (D) $^{e}$
}

The Prins cyclization of styrene (SE) with paraformaldehyde (PFCHO) was conducted with mesoporous ZnAlMCM-41 catalysts for the synthesis of 4-phenyl-1,3-dioxane (4-PDO) using a liquid phase heterogeneous catalytic method. For a comparison study, the Prins cyclization reaction was also conducted over different nanoporous catalysts, e.g. mesoporous solid acid catalysts, AIMCM-41(21) and ZnMCM-41 (21), and microporous catalysts, USY, H $\beta, \mathrm{HZSM}-5$, and $\mathrm{H}$-mordenite. The recyclable mesoporous ZnAIMCM-41 catalysts were reused in this reaction to evaluate their catalytic stabilities. Since ZnAIMCM-41(75) has higher catalytic activity than other solid acid catalysts, washed ZnAIMCM-41(75)/ W-ZnAIMCM-41(75) was prepared using an efficient chemical treatment method and used with various reaction parameters to find an optimal parameter for the highly selective synthesis of 4-PDO. W-ZnAIMCM-41(75) was also used in the Prins cyclization of olefins with PFCHO and formalin (FN, 37\% aqueous solution of formaldehyde (FCHO)) under different reaction conditions to obtain 1,3-dioxanes, which are widely used as solvents or intermediates in organic synthesis. Based on the nature of catalysts used under different reaction conditions, a reasonable plausible reaction mechanism for the Prins cyclization of SE with PFCHO is proposed. Notably, it can be seen from the catalytic results of all catalysts that the W-ZnAlMCM-41(75) catalyst has higher 4-PDO selectivity with exceptional catalytic activity than other microporous and mesoporous catalysts.

\section{Introduction}

The addition of aldehydes and ketones to alkenes in the presence of Brønsted acids is usually called the Prins reaction, which is a straightforward catalyst method for the synthesis of 1,3-dioxanes using a variety of olefins with FCHO in the presence of sulfuric acid $\left(\mathrm{H}_{2} \mathrm{SO}_{4}\right)$, which is called a mineral Brønsted acid catalyst, under a homogeneous medium, and in acid-catalyzed condensation reactions, water and glacial acetic acid were used as solvents. ${ }^{1,2}$ Citronellal to isopulegol is one of the Prins cyclization reactions. ${ }^{2}$ In this reaction, many

\footnotetext{
${ }^{a}$ Department of Chemistry, Faculty of Science, King Khalid University, Abha 61413, Saudi Arabia. E-mail: mselvaraj@kku.edu.sa; Fax: +966172417637

${ }^{b}$ Department of Chemistry, School of Basic and Applied Sciences, RIMT University, Mandi Gobindgarh, Punjab, 147301, India

${ }^{c}$ School of Chemical Sciences, Universiti Sains Malaysia, 11800 Penang, Malaysia

${ }^{d}$ Department of Chemistry, Indian Institute of Technology Hyderabad, Kandi,

Sangareddy, Telangana, India

${ }^{e}$ Department of Polymer Science and Engineering, Pusan National University,

Busan 609-735, Republic of Korea

$\dagger$ Electronic supplementary information (ESI) available. See DOI: 10.1039/ dodt04158k
}

Brønsted acids and Lewis acids, e.g., phosphoric acid $\left(\mathrm{H}_{3} \mathrm{PO}_{4}\right)$, acetic acid $\left(\mathrm{CH}_{3} \mathrm{COOH}\right)$, boron trifluoride $\left(\mathrm{BF}_{3}\right)$ and stannic chloride $\left(\mathrm{SnCl}_{4}\right)$, were used as catalysts. ${ }^{3-5}$ The mineral Brønsted and Lewis acid catalysts used in homogeneous catalytic systems can easily damage the reactors because they cause high corrosion and excessive toxic wastes. The recovery of identical catalysts takes a long time after the reaction is complete, most undesirable side products are likely formed by the use of large quantities of raw chemicals, and many steps are needed to recover them from the main product. A major drawback is that homogeneous catalysts cannot be used as recyclable catalysts in the chemical industry and are considered as non-ecofriendly.

In order to overcome the above problems, the heterogeneous catalytic chemistry mainly involves the development of a highly efficient and versatile catalyst system for producing 1,3-dioxanes through Prins cyclization reactions. Moreover, the heterogeneous catalytic system produces the main product with high selectivity, reduces the trace number of byproducts and replaces hazardous and stoichiometric reagents, in which the ecofriendly catalytic materials are easily recovered from the final reaction mixture and used as recyclable catalysts. Recent 
research has focused on replacing traditional homogeneous catalysts with green heterogeneous catalysts. For the Prins cyclization reaction of olefins, several heterogeneous solid acid catalysts have been designed and developed, e.g., zeolites, organic heteropolysalts, sulfonic group functionalized SBA-15 $\mathrm{MoO}_{3} / \mathrm{SiO}_{2}$ and sulfated zirconia. ${ }^{6-11}$ Using the above catalysts, 1,3-dioxane derivatives synthesized by the Prins cyclization of olefins with PFCHO/FN are used in industry as solvents, plasticizers, curing agents and pigment dispersants, and as intermediates in the preparation of pharmaceuticals in medicinal organic synthetic chemistry. ${ }^{6-13}$

Corporate pressure to reduce the amount of toxic waste arising from environmental protection laws and chemical processes has fostered the development of innovative and environmentally friendly chemical technologies. For application in fine chemical industries, suitable solid acid catalysts are in high demand, synthesized with a large number of catalytically active sites, and used under mild reaction conditions without the use of toxic materials. For the green catalytic process by reducing environmental pollution, Selvaraj's research group, for the first time, developed hexagonally mesostructured ZnAlMCM-41 catalysts with good physiochemical properties. ${ }^{14}$ ZnAlMCM-41catalysts have been widely used for the selective synthesis of nopol through the Prins condensation reaction. ${ }^{15}$ To the best of our knowledge, ZnAlMCM-41 catalysts have not been used for the Prins cyclization of olefins with PFCHO/FN, and their catalytic results have not been clearly reported in the open literature. Our present investigation brings out a unique, eco-friendly and simple method for the selective synthesis of 4-PDO and different 1,3 dioxanes using low cost reactants under a liquid phase catalytic system while true heterogeneous catalysts, ZnAlMCM-41, have been recently used in this catalyst system. The catalytic results obtained from PFCHO and FN using ZnAlMCM-41 catalysts were thoroughly discussed to find the best one. The catalytic results obtained over ZnAlMCM-41 catalysts, for the selective synthesis of 1,3-dioxanes, have also been correlated and compared with those of other nanoporous solid catalysts, such as AlMCM-41(21), ZnMCM-41(21), USY, $\mathrm{H} \beta$, HZSM-5 and H-mordenite.

\section{Experimental}

According to previously published procedures, ${ }^{14,16,17}$ various mesoporous catalysts ((ZnAlMCM-41, $n_{\mathrm{Si}} /\left(n_{\mathrm{Zn}}+n_{\mathrm{Al}}\right)=75,151$, 228, 304 and 380), (AlMCM-41, $\left.n_{\mathrm{Si}} / n_{\mathrm{Al}}=21\right)$, (ZnMCM-41, $n_{\mathrm{Si}} /$ $n_{\mathrm{Zn}}=21$ ), and MCM-41) were synthesized using a direct hydrothermal method and characterized. The discussion of physicochemical characterization and the details of the synthesis procedures of mesoporous catalysts are given in the ESI. $\uparrow$ Microporous catalysts, $\mathrm{H} \beta\left(n_{\mathrm{Si}} / n_{\mathrm{Al}}=20\right.$, Strem $), \mathrm{HY}\left(n_{\mathrm{Si}} / n_{\mathrm{Al}}=\right.$ 2.9, PQ), H-mordenite $\left(n_{\mathrm{Si}} / n_{\mathrm{Al}}=20, \mathrm{PQ}\right)$, and HZSM-5 $\left(n_{\mathrm{Si}} / n_{\mathrm{Al}}=\right.$ $30, \mathrm{PQ})$, were obtained as commercial sources. Prior to the use of catalytic materials in this catalytic reaction, they were calcined at $473 \mathrm{~K}$ in air for $6 \mathrm{~h}$ to completely remove moisture from their inner pore surfaces.
Prins cyclization of SE with $\mathrm{PFCHO}$ was conducted in a liquid phase reaction using different nanoporous catalysts under vigorous stirring in a thermostated quartz-vessel reactor. In a typical experimental procedure, $100 \mathrm{mg}$ of ZnAlMCM-41 (75) was placed in the reactor with the required amounts of SE (20 mmol), PFCHO (20 mmol) and 1,2-dichloroethane solvent (5 mL). The reactor fitted with a condenser was slowly heated to $353 \mathrm{~K}$ with continuous stirring. After reaching $353 \mathrm{~K}$, the reaction mixture was continuously refluxed for $6 \mathrm{~h}$ under atmospheric pressure. After completion of the reaction, the products were collected through the recovery of ZnAlMCM-41 (75). The products cooled to room temperature were centrifuged, analyzed with authentic samples using a gas chromatography system (GC, Shimadzu-2041, FID detector) equipped with an RTX-5 column (0.25 mm I.D. and $30 \mathrm{~m}$ length). All the products were further confirmed using a combined gas chromatography-mass spectrometry system (GC-MS; Hewlett G1800A).

To find the best catalyst, various nanoporous catalysts were used for the Prins cyclization of SE with PFCHO under similar reaction conditions. The studies of recyclable and washed catalysts were performed. The Prins cyclization of SE with PFCHO was also performed with different solvents to identify the best solvent. Various reaction parameters, i.e. the temperature, time and stoichiometric ratio of the reactants (SE to PFCHO ratios), were studied to determine the optimal reaction conditions. For the production of 4-PDO, FN was also used instead of PFCHO. The Prins cyclization of olefins with PFCHO was also conducted with different reaction times and temperatures under similar reaction conditions. The organic products were extracted after completion of the reaction, cooled to room temperature, and analysed with authentic samples by GC and further confirmed by GC-MS. The olefin conversion, product yield and selectivity were calculated using the standard formulas. The NMR $\left({ }^{1} \mathrm{H}\right.$ and $\left.{ }^{13} \mathrm{C}\right)$ data for the selected 1,3-dioxane products are specified in the ESI. $\dagger$

\section{Results and discussion}

\subsection{Selective synthesis of 4-PDO}

The Prins cyclization of SE with PFCHO was carried out over different nanoporous solid catalysts with a corresponding ratio of $1: 2$ at $353 \mathrm{~K}$ for $6 \mathrm{~h}$, as noted in Table 1 . In this cyclization reaction, 4-PDO formed as a major product by cycloaddition of the primary product intermediate, 3-phenylpropanol-like cation, with another FCHO molecule being derived from PFCHO, as illustrated in Scheme 1.

The sulfonic acid functionalized SBA-15 (SBA-15- $\left.\mathrm{SO}_{3} \mathrm{H}\right)$ produced $100 \%$ 4-PDO selectivity when the Prins cyclization was conducted with SE and FCHO at a molar ratio of $1: 2$ with dichloromethane as a solvent in refluxing temperature for $35 \mathrm{~h}$ in a liquid phase reaction but catalyzed to 52\% 4-PDO selectivity only when water was used as a solvent under similar reaction conditions. ${ }^{7}$ The catalytic results show that $\mathrm{SBA}-15-\mathrm{SO}_{3} \mathrm{H}$ has large numbers of free silanol groups, which reduce hydrophobicity and cause the migration of acid sites, indicating that 
Table 1 Prins cyclization of SE with PFCHO and FN over various types of catalysts ${ }^{a}$

\begin{tabular}{|c|c|c|c|c|}
\hline Catalysts (100 mg) & Pore size $(\AA)$ & Conversion of SE (\%) & Selectivity of 4-PDO (\%) & Yield of 4-PDO (\%) \\
\hline ZnAlMCM-41(151) & 25.6 & 91 & 99 & 90 \\
\hline ZnAlMCM-41(304) & 29.3 & 75 & 93 & 70 \\
\hline ZnAlMCM-41(380) & 32.2 & 67 & 90 & 60 \\
\hline HY (2.6) & 7.4 & 60 & 49 & 29 \\
\hline $\mathrm{H} \beta(20)$ & $7.6 \times 5.4$ & 64 & 79 & 51 \\
\hline AlMCM-41(21) & 27.5 & 85 & 95 & 81 \\
\hline ZnMCM-41(21) & 27.3 & 40 & 81 & 32 \\
\hline MCM-41 & 29.3 & 11 & 38 & 4 \\
\hline Without catalyst & - & 9 & 33 & 3 \\
\hline ZnAlMCM-41(75) $)^{b}$ & 22.8 & 99 & 100 & 99 \\
\hline ZnAlMCM-41(75) ${ }^{c}$ & 22.8 & 100 & 100 & 100 \\
\hline${\text { ZnAlMCM-41 }(75)^{d}}^{d}$ & 22.8 & 97 & 100 & 97 \\
\hline
\end{tabular}

${ }^{a}$ Reaction conditions: $10 \mathrm{mmol}$ of SE, $20 \mathrm{mmol}$ of PFCHO, $5 \mathrm{ml}$ of 1,2-dichloroethane, temperature $=353 \mathrm{~K}$, time $=6 \mathrm{~h} .{ }^{b} 30 \mathrm{mmol}$ of $\mathrm{FN}(37 \%$ aqueous solution of FCHO) instead of $20 \mathrm{mmol}$ of PFCHO. ${ }^{c}$ The reaction was carried out with PFCHO at $413 \mathrm{~K}$ in an autoclave under autogenous pressure for 3 h. ${ }^{d} 30 \mathrm{mmol}$ of $\mathrm{FN}$ without solvent. ${ }^{e}$ The reaction was carried out using $30 \mathrm{mmol}$ of $\mathrm{FN}$ at $413 \mathrm{~K}$ in an autoclave under autogenous pressure for $3 \mathrm{~h}$.

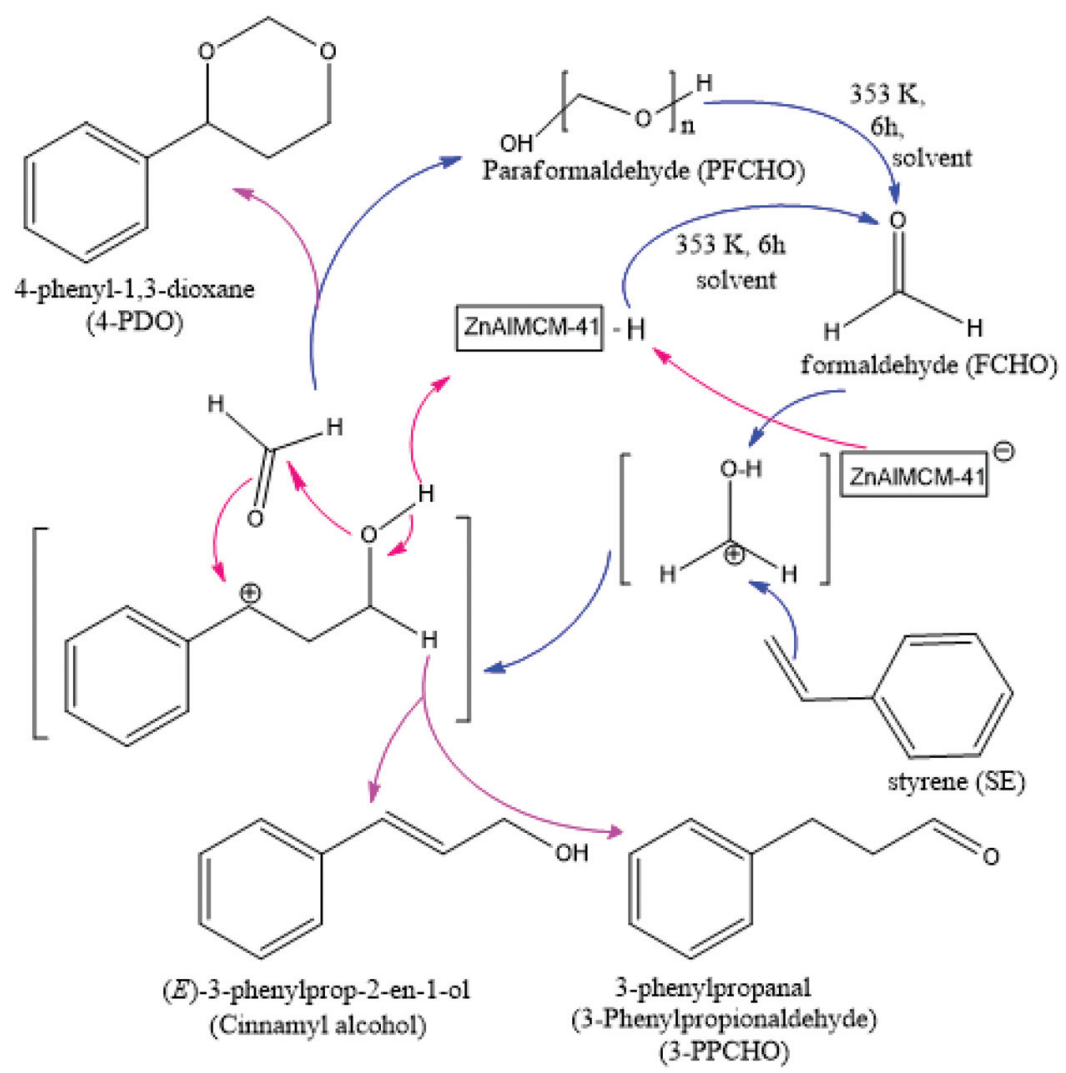

Scheme 1 Plausible reaction mechanism for the Prins cyclization of SE with PFCHO over ZnAIMCM-41 catalysts.

FCHO is an inappropriate source for the Prins cyclization of $\mathrm{SE}$ over $\mathrm{SBA}-15-\mathrm{SO}_{3} \mathrm{H}$. The sulfated zirconia (SZ) catalyst was used in the Prins cyclization with SE and PFCHO at a molar ratio of $1: 3$ at $353 \mathrm{~K}$ for $7 \mathrm{~h}$ and gave 92\% 4-PDO selectivity when 1,2-dichlorethane was used as a solvent. Although the SZ catalyst produces a better 4-PDO selectivity, the characteristic results show that the surface area of $\mathrm{SZ}$ is fourteen times less than that of the ZnAlMCM-41(75) catalyst whereas the SZ cata- 
lyst has different pore sizes, which enhance the production of polymerized products with $8 \%$ selectivity. ${ }^{11}$ The Wells-Dawson type heteropolyacid $\left(\mathrm{H}_{6} \mathrm{P}_{2} \mathrm{~W}_{18} \mathrm{O}_{62} \cdot 24 \mathrm{H}_{2} \mathrm{O}\right)$ catalyst yielded $68 \%$ selectivity with $4.8 \% \mathrm{SE}$ conversion at $298 \mathrm{~K}$ when the Prins cyclization of SE was conducted with FCHO using water as a solvent. ${ }^{9}$ This result indicates that the heteropolyacid catalyst has high hydrophilicity with a low surface area, and even it has more strong acid sites compared to solid acid catalysts while water molecules inhibit its acid sites during the production of 4-PDO. Although the triflic acid (TfOH) catalyst gives a $92 \%$ yield of 4 -PDO at $353 \mathrm{~K}$ for $10 \mathrm{~h}$ during the Prins cyclization of SE with $\mathrm{FCHO}$, it is a non-ecofriendly catalyst due to the highly corrosive and eye irritant liquid and it cannot be easily reused. ${ }^{18}$ To overcome these disadvantages, mesoporous ZnAlMCM-41 catalysts were used in Prins cyclization, and they showed excellent 4-PDO selectivity. This is due to the $\mathrm{Zn}$ ions incorporated into mesoporous AlMCM-41 catalysts, which are very favorable for cyclization, condensation, hydrogenation and dehydrogenation, as reported elsewhere. ${ }^{16}$ The yield of 4-PDO, as shown in Table 1, increases in the order of ZnAlMCM-41(75) > ZnAlMCM-41(151) > AlMCM-41(21) $\approx$ ZnAlMCM-41(228) > ZnAlMCM-41(304) > ZnAlMCM-41(380) > $\mathrm{H} \beta(20)>$ ZnMCM-41(21) > $\mathrm{HY}(2.6)>\mathrm{H}$-mordenite $(20)>$ HZSM-5(15) > MCM-41. Mesoporous ZnAlMCM-41(75) has higher SE conversion (100\%) and 4-PDO selectivity (100\%) as well as $100 \%$ yield of 4 -PDO compared to other mesoporous and microporous catalysts (Table 1) and shows excellent catalytic activity that enhances the 4-PDO selectivity because it does not have any diffusional constraints. While the results of the catalytic activity prove that ZnAlMCM-41(75) has a larger pore size than the molecular size of 4-PDO as reported elsewhere. ${ }^{16}$ Due to an increase in the amounts of $\mathrm{Zn}$ and $\mathrm{Al}$ ions, as shown in Tables $1 \mathrm{~S}$ and $2 \mathrm{~S} \dagger$, ZnAlMCM-41(75) has higher Brønsted acid sites with a less pore size compared to other ZnAlMCM-41 catalysts while its acidity enhances the 4-PDO selectivity compared to other ZnAlMCM-41 catalysts. Additionally, within $6 \mathrm{~h}$, the ZnAlMCM-41(75) catalyst improves the selectivity of 4-PDO compared to other catalysts e.g. SZ and TfOH, as observed in the literature. ${ }^{11,18}$ In this catalytic reaction, the selectivity $(100 \%)$ of $4-\mathrm{PDO}$ remains constant when FN is used instead of PFCHO/when the catalytic reaction is carried out with PFCHO at $413 \mathrm{~K}$ for $3 \mathrm{~h}$ under autogenous pressure/when FN is used without solvent/when the Prins cyclization of SE is conducted with FN at $413 \mathrm{~K}$ for $3 \mathrm{~h}$ under autogenous pressure. All catalytic studies have shown that a large number of Brønsted acid sites in ZnAlMCM-41(75) can be promoted by the introduction of large amounts of $\mathrm{Zn}$ ions onto the surface of mesoporous AlMCM-41, which has been confirmed by FTIR-pyridine-acidity studies. ${ }^{14}$ In addition, we found from the acidity studies of FTIR-pyridine and TPD-pyridine that the total acidity, and Brønsted and Lewis acid sites increase with an increase in the amount of $\mathrm{Zn}$-ions incorporated on the surface of AlMCM-41 as reported in Table $2 \mathrm{~S}, \dagger$ while ZnAlMCM-41(75) has high hydrophobicity, which is confirmed by the study of its hydrothermal stability. ${ }^{14}$ The $\mathrm{H} \beta(20)$ catalyst produces a higher yield of 4-PDO with better selectivity than other porous catalysts, as depicted in Table 1, because its mild acid sites are more suited to this catalytic system than other catalysts, as revealed elsewhere. ${ }^{8}$ ZnMCM-41(21) produces a lower yield of 4-PDO with $81 \%$ selectivity than other mesoporous catalysts but increases the selectivities of the byproducts, e.g. cinnamyl alcohol (15\%) and 3-phenylpropionaldehyde (4\%, 3-PPCHO). This catalytic result shows that the acid sites of ZnMCM-41(21) could not favor the Prins cyclization reaction, as revealed in the literature. ${ }^{19}$ Based on the literature results, the results of FT-IR-pyridine and ammoniaTPD indicate that the acid sites of all microporous catalysts are almost identical, except for ZSM-5, as confirmed elsewhere. ${ }^{20,21}$ Even though zeolite $\mathrm{H} \beta(20)$ with pore sizes of 7.6 and $5.4 \AA$ has fewer acid sites than $\operatorname{HY}(2.6)$ due to a lower aluminum content, it produces a higher yield of 4-PDO with $64 \%$ selectivity than $\mathrm{HY}(2.6)$. This catalytic result implies that the pore size $(7.4 \AA)$ of $\mathrm{HY}(2.6)$ could not enhance in the production of 4-PDO but it rather increases the selectivity of cinnamyl alcohol $(40 \%)$, as shown in Table 1 . These catalytic results clearly demonstrate that both catalysts (H $\beta$ and $\mathrm{HY}$ ) cannot generate high yields of 4-PDO in their inner pores due to diffusion limit constraints for 4-PDO; furthermore, large numbers of reactants and large organic molecules cannot normally enter into their inner pores as commonly reported elsewhere. ${ }^{21}$ In contrast, H-mordenite(20) and HZSM-5(15) are less active in the reaction because aryl-1,3-dioxanes are too large and cannot enter into their pores (ZSM-5 (5.4 $⿱$ ) $)$ and H-mordenite $(7.1 \AA))$ ). Hence, 4-PDO forms only on the outer surfaces of ZSM-5 and H-mordenite, as referred to in the literature. ${ }^{8}$ Although this reaction is catalyzed by moderate active sites, H-mordenite(20) produces a higher yield of 4-PDO with 43\% selectivity than HZSM-5(15). These catalytic results show that the high-acid sites and small pore-sized zeolites of ZSM-5 do not require the Prins cyclization reaction, as mentioned in Table 1. Overall, this is corroborated from all the above catalytic results, as all the mesoporous catalysts, viz. ZnAlMCM-41 and AlMCM-41(21), with the exception of ZnMCM-41(21), produce higher yields of 4-PDO with exceptional selectivity compared to all the microporous catalysts, which are not suitable for this reaction to achieve a higher yield of 4-PDO. Furthermore, ZnAlMCM-41(75) proves to be an excellent catalyst for the highly selective synthesis of 4-PDO. In conclusion, it confirms that the highly ordered mesopore with large amounts of $\mathrm{Zn}$ and $\mathrm{Al}$ loaded into MCM-41 plays an important catalytic role in the obtainment of high 4-PDO selectivity.

\subsection{Recyclability}

Of the mesoporous catalysts used in the Prins cyclization reaction, ZnAlMCM-41(75) and ZnAlMCM-41(380) produce the highest and lowest 4-PDO selectivities, respectively. After the investigation of the above catalytic results, the reusabilities of the mesoporous catalysts, ZnAlMCM-41(75) and ZnAlMCM-41 (380), were mainly evaluated to investigate their catalytic stabilities under the reaction conditions specified in Table 2 . Initially, the catalysts used for this reaction suffered from loss of catalytic activity and cannot be directly reused because trace 
Table 2 Recycling of ZnAIMCM-41(75) and ZnAlMCM-41(380) for Prins cyclization of SE with PFCHO

\begin{tabular}{|c|c|c|c|c|c|}
\hline Catalysts (100 mg) & Pore size $(\AA)$ & Run & Conversion of SE (\%) & Selectivity of 4-PDO (\%) & Yield of 4-PDO (\%) \\
\hline ZnAlMCM-41(75) ${ }^{b}$ & 22.7 & - & 90 & 92 & 83 \\
\hline ZnAlMCM-41 $(75)^{c}$ & 22.8 & 1 & 99 & 100 & 99 \\
\hline ZnAlMCM-41(75) ${ }^{c}$ & 22.9 & 2 & 100 & 100 & 100 \\
\hline ZnAlMCM-41(75) & 22.9 & 3 & 100 & 100 & 100 \\
\hline ZnAlMCM-41(75) ${ }^{c}$ & 22.9 & 4 & 100 & 100 & 100 \\
\hline W-ZnAlMCM-41(75) ${ }^{d}$ & 22.9 & - & 100 & 100 & 100 \\
\hline ZnAlMCM-41 $(380)^{c}$ & 31.5 & 1 & 65 & 88 & 57 \\
\hline ZnAlMCM- $41(380)^{c}$ & 31.5 & 2 & 66 & 89 & 59 \\
\hline ZnAlMCM-41(380) ${ }^{c}$ & 31.5 & 3 & 67 & 90 & 60 \\
\hline ZnAlMCM- $41(380)^{c}$ & 31.5 & 4 & 67 & 90 & 60 \\
\hline
\end{tabular}

${ }^{a}$ Reaction conditions: $10 \mathrm{mmol}$ of SE, $20 \mathrm{mmol}$ of PFCHO, $5 \mathrm{ml}$ of 1,2-dichloroethane, temperature $=353 \mathrm{~K}$, time $=6 \mathrm{~h} .{ }^{b}$ The used catalyst was directly used (no chemical treatment/calcinations). ${ }^{c}$ The reused catalysts were obtained, as the used catalysts were chemically treated with acetone several times, dried at $393 \mathrm{~K}$ overnight and calcined at $773 \mathrm{~K}$ for $6 \mathrm{~h}$ in air to remove the unwanted organics completely. ${ }^{d}$ The catalyst was washed with ammonium chloride solution for removal of non-framework zinc and aluminium ions on the active surface.

amounts of unreacted organic coke accumulated on the surface of the catalysts. Therefore, the catalysts were regenerated by chemical treatments and calcinations before use. An experimental procedure for a clear observation is illustrated. To remove the unwanted organics completely, the reused catalyst was chemically treated with acetone several times, dried at $393 \mathrm{~K}$ overnight and calcined at $773 \mathrm{~K}$ for $6 \mathrm{~h}$ in air. Under the same experimental procedure, all recyclable catalysts were recovered before each successive run. The conversion of SE and selectivity, and the yield of 4-PDO slightly decrease in the first two runs. These results indicate that trace amounts of non-framework octahedral aluminum and zinc oxides accumulated on the inner pore surface of ZnAlMCM-41(75) screen some active sites and can be completely removed using the above procedure. After two runs, the catalysts in cycles without losing their activities on the surface of the mesoporous silica matrix remain unchanged with the conversion of SE and selectivity as well as the yield of 4-PDO. This result is corroborated by the results of ICP-AES obtained before/after the reuse of ZnAlMCM-41(75), as depicted in Table $1 \mathrm{~S} . \dagger$ The catalytic results of each recycle show that ZnAlMCM-41(380) predicts nearly identical catalytic activity given in Table 2 because low active species are homogenously incorporated in the inner silica pore walls. This process is a truly heterogeneous catalytic process. ${ }^{21}$ To ensure the catalytic stability with the results of the recycling experiments, ZnAlMCM-41(75) was simply washed three times with $1.0 \mathrm{M} \mathrm{NH} \mathrm{NH}_{4} \mathrm{Cl}$ aqueous solution (10 mg of ZnAlMCM-41/20 mL of $1.0 \mathrm{M} \mathrm{NH} \mathrm{NH}_{4} \mathrm{Cl}$ solution) at room temperature for $1 \mathrm{~h}$, filtered, washed with copious amounts of deionized water, and air-dried at $373 \mathrm{~K}$ for $1 \mathrm{~h}$. The treated catalyst is denoted as W-ZnAlMCM-41(75), which was used in the preparation of 4-PDO. The yield of 4-PDO for W-ZnAlMCM-41(75) is similar to that of the third cycle obtained for recyclable ZnAlMCM-41(75). This catalytic statement confirms the catalytic stability because the acid sites are almost unchanged from the surface of the two catalysts. The pure mesoporous silica, MCM-41 was used in this reaction but gave the lowest SE conversion (11\%) with the trace yield of 4-PDO (Table 1). The conversion of SE is much less when the reaction is conducted without a catalyst (Table 1 ). In conclusion, the major catalytically active sites are created only by both the metal ions $\mathrm{Al}$ and $\mathrm{Zn}$ incorporated/substituted on the surface of silica walls.

\subsection{Effect of solvents}

Since W-ZnAlMCM-41(75) gives excellent SE conversion and yield as well as selectivity of 4-PDO among the other nanoporous catalysts, it is used to find the best solvent for the highly selective synthesis of 4-PDO through Prins cyclization under the reaction conditions specified in Table 3. The Prins cyclization of SE with PFCHO was conducted with non-polar, polar aprotic and polar protic solvents. Initially, the different types of solvents with nearly identical dielectric constants (DCs) used in this reaction show different yields for 4-PDO and SE conversions as mentioned in Table 3. For example, it is interesting to note that the yield of 4-PDO is different in solvents with identical DCs, e.g., toluene (DC 2.38), cyclohexane (DC 2.02), triethylamine (DC 2.42) and 1,4-dioxane (2.25), as shown in Table 3 . These catalytic results demonstrate that DC is not an important factor in evaluating the catalytic activity of ZnAlMCM-41(75) by the Prins cyclization of SE with PFCHO.

The above results decide further to study the Acceptor number (AN) and Donor number (DN) of solvents used in the Prins cyclization reaction when PFCHO is used as the FCHO source, as noted in Table 3. Solvent properties such as DN and AN clearly illustrate the role of solvents in this catalytic reaction. Gutman first reported the properties of DN and AN of different solvents by investigating their acidic and basic nature. ${ }^{22}$ Furthermore, many chemists working in academia and industry have adopted the Gutman concepts to evaluate the impacts of solvents on this reaction using a liquid phase reaction method. ${ }^{23,24}$ As shown in Table 3, the yield of 4-PDO increases when the AN of the solvents is higher than the DN but it decreases when the DN of the solvents is higher than the AN. However, dichloromethane, which is a volatile and polar aprotic solvent and has a better AN, produces $29 \%$ yield of 4 -PDO with $45 \%$ SE conversion only because it could not 
Table 3 Prins cyclization of SE with PFCHO and FN using different solvents over W-ZnAlMCM-41(75)

\begin{tabular}{|c|c|c|c|c|c|c|c|c|}
\hline Catalysts & $\begin{array}{l}\text { Boiling } \\
\text { point }\left({ }^{\circ} \mathrm{C}\right)\end{array}$ & $\begin{array}{l}\text { Dipole } \\
\text { moment (D) }\end{array}$ & $\begin{array}{l}\text { Dielectric } \\
\text { constant (DC) }\end{array}$ & $\begin{array}{l}\text { Acceptor } \\
\text { number (AN) }\end{array}$ & $\begin{array}{l}\text { Donor number } \\
(\mathrm{DN})\end{array}$ & $\begin{array}{l}\text { Conversion } \\
\text { of SE }(\%)\end{array}$ & $\begin{array}{l}\text { Selectivity of } \\
4 \text {-PDO }(\%)\end{array}$ & $\begin{array}{l}\text { Yield of } \\
4 \text {-PDO }(\%)\end{array}$ \\
\hline Dichloromethane & 39.6 & 1.55 & 8.93 & 20.4 & 1 & 45 & 65 & 29 \\
\hline Chloroform & 61.2 & 1.15 & 4.81 & 23.1 & 4 & 75 & 83 & 62 \\
\hline 1,2-Dichloroethane & 55 & 1.86 & 10.36 & 16.7 & 0 & 100 & 100 & 100 \\
\hline Toluene & 110.6 & 0.43 & 2.38 & 8.2 & 0 & 85 & 88 & 75 \\
\hline Cyclohexane & 80.75 & 0 & 2.02 & 0 & 0 & 15 & 65 & 10 \\
\hline Tetrahydrofuran & 66 & 1.73 & 7.58 & 8.0 & 20 & 14 & 53 & 7 \\
\hline Dimethylformamide & 153 & 3.86 & 36.7 & 16.0 & 26.6 & 16 & 58 & 9 \\
\hline Dimethylactamide & 165 & 3.72 & 37.8 & 13.6 & 27.8 & 18 & 49 & 9 \\
\hline Acetonitrile & 82 & 3.45 & 37.5 & 18.9 & 14.1 & 23 & 69 & 16 \\
\hline Nitrobenzene & 210 & 4.02 & 34.82 & 14.8 & 4.4 & 61 & 72 & 44 \\
\hline Triethylamine & 89 & 0.87 & 2.42 & 1.4 & 61 & 10 & 28 & 3 \\
\hline Isopropylalcohol & 82.5 & 1.66 & 17.9 & 33.5 & 21.1 & 39 & 52 & 20 \\
\hline 1,4-Dioxane & 101 & 0.45 & 2.25 & 10.8 & 0.38 & 96 & 98 & 94 \\
\hline Water & 100 & 1.82 & 80.1 & 54.8 & 18 & 92 & 100 & 92 \\
\hline Without solvent & - & - & - & - & - & 30 & 47 & 14 \\
\hline 1,4-Dioxane ${ }^{b}$ & 101 & 0.45 & 2.25 & 10.8 & 0.38 & 98 & 100 & 98 \\
\hline Dichloromethane $^{b}$ & 39.6 & 1.55 & 8.93 & 20.4 & 1 & 100 & 98 & 98 \\
\hline Water $^{b}$ & 100 & 1.82 & 80.1 & 54.8 & 18 & 97 & 100 & 97 \\
\hline Without solvent ${ }^{b}$ & - & - & - & - & - & 91 & 100 & 91 \\
\hline
\end{tabular}

${ }^{a}$ Reaction conditions: $100 \mathrm{mg}$ of W-ZnAlMCM-41(75), $10 \mathrm{mmol}$ of SE, $20 \mathrm{mmol}$ of PFCHO, $5 \mathrm{ml}$ of solvent, temperature $=353 \mathrm{~K}$, time $=6 \mathrm{~h}$. ${ }^{b} 30 \mathrm{mmol}$ of $\mathrm{FN}$ instead of $20 \mathrm{mmol}$ of PFCHO.

achieve the complete depolymerization of PFCHO in the formation of FCHO. Nitrogen containing solvents are unable to produce a better yield of 4-PDO because their DNs are higher than ANs, except nitrobenzene, which produces $44 \%$ yield of 4-PDO because it has a higher AN compared to the DN. It is noteworthy from the above catalytic results that solvents with high ANs and low DNs show higher selectivity of 4-PDO. For clear information, water has a higher AN compared to the DN and shows higher selectivity of 4-PDO. This result indicates that the hydrophobicity of ZnAlMCM-41(75) leads to higher catalytic activity as confirmed elsewhere. ${ }^{14}$ The complete depolymerization of PFCHO to form FCHO over ZnAlMCM-41(75) occurs when a solvent with a high AN is used in this reaction. While the solvent represents the Lewis acidic property, it enables the formation of FCHO from PFCHO without the impacts of catalytically active sites. The nitrogen-containing solvents (Table 3) with high DNs make poison-treatment on the acid sites of ZnAlMCM-41(75) and reduce the active sites, while the solvents reduce the formation of FCHO from PFCHO and the yield of 4-PDO because they are Lewis basic in nature, which tends to coordinate with Lewis acceptors with various interactions. ${ }^{25}$ Isopropyl alcohol is one of the protic solvents, has a higher DN compared to non-nitrogen containing solvents and could not help to improve the catalytic activity. The catalytic reaction conducted without a solvent produces $14 \%$ yield of 4-PDO. Overall, to produce $100 \%$ yield of 4-PDO with $100 \%$ SE conversion, 1,2-dichloroethane, which has a high AN and zero DN, is a good solvent compared to other solvents for the Prins cyclization of SE with PFCHO.

To evaluate the solvent effect for the highly selective synthesis of 4-PDO, the FN was used as an FCHO source for the Prins cyclization of SE over W-ZnAlMCM-41(75) with different solvents, viz. 1,4-dioxane (non-polar), dichloromethane (non- polar) and water (polar protic), as specified in Table 3. Dichloromethane plays a unique role in obtaining $98 \%$ yield of 4-PDO with $100 \%$ SE conversion. The other non-polar solvent, 1,4-dioxane, yields almost the same catalytic activity as mentioned in Table 3. High catalytic activity (91\% SE conversion and $100 \%$ 4-PDO selectivity) is observed when the reaction is carried out in the absence of a solvent. The catalytic results indicate that when $\mathrm{FN}$ is used instead of PFCHO, an organic solvent is not required to produce high yields of 4-PDO because the water content of FN acts as a solvent. For additional information, the catalytic reaction when using water as a solvent provides 100\% 4-PDO selectivity with 97\% SE conversion. In this reaction, ZnAlMCM-41(75) possesses higher catalytic activity than other solid acid catalysts. ${ }^{7,9}$ The results of the above catalyst demonstrate that catalytically active sites cannot be excreted from the surface of W-ZnAlMCM-41(75) because it has high hydrophobicity with improved hydrothermal stability. ${ }^{14}$ Moreover, FN and water are the most attractive substrate and solvent, respectively, and are used in fine chemical industries to produce high quantities of 4-PDO with high yields, as reported elsewhere. ${ }^{7}$ Overall, water is a green solvent, inexpensive and readily available for the highly selective synthesis of 4-PDO by the Prins cyclization of SE with FN being used as the FCHO source only while this type of synthesis procedure is considered as a green heterogeneous catalytic method.

\subsection{Effect of reaction parameters}

The Prins cyclization of SE with PFCHO was performed with different reaction temperatures using W-ZnAlMCM-41(75) under the reaction conditions specified in Fig. 1. As the temperature increases from 293 to $353 \mathrm{~K}$, the SE conversion with selectivity and the yield of 4-PDO reach 100\% within 6 hours. 


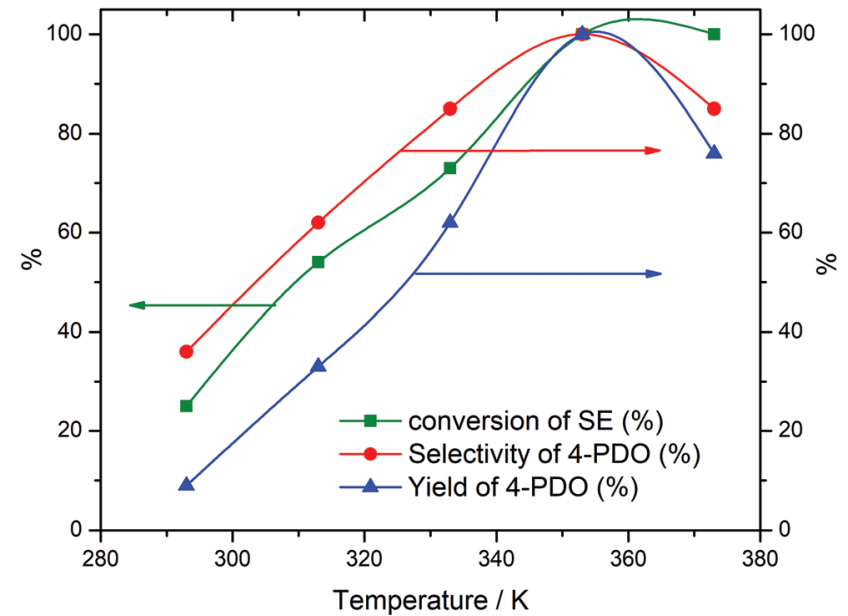

Fig. 1 Effect of reaction temperature over W-ZnAIMCM-41(75). Reaction conditions: $100 \mathrm{mg}$ of catalyst, $10 \mathrm{mmol}$ of SE, $20 \mathrm{mmol}$ of $\mathrm{PFCHO}, 5 \mathrm{ml}$ of 1,2-dichloroethane, time $=6 \mathrm{~h}$, SE to PFCHO mole ratio $=1: 2$.

This is because the depolymerization of PFCHO is very fast in the formation of FCHO; while a complete SE conversion is observed within 6 hours. The main product, 4-PDO, selectivity gradually decreases to $\sim 85 \%$ but the $\mathrm{SE}$ conversion remains constant when the reaction is carried out at above $353 \mathrm{~K}$ because the formation of byproducts could be observed (not shown in Fig. 1). From the literature, it is noteworthy that W-ZnAlMCM-41(75) has $100 \%$ catalytic activity at $353 \mathrm{~K}$ than other solid acid catalysts. ${ }^{9,11}$ The aforementioned catalytic results demonstrate that the Prins cyclization reaction continues to exhibit better conversion and selectivity at $353 \mathrm{~K}$ than other reaction temperatures.

To find the best selective synthesis of 4-PDO, the Prins cyclization of SE with PFCHO is carried out at different reaction times over W-ZnAlMCM-41(75) under the reaction conditions shown in Fig. 2. An increase in conversion from 55 to $100 \%$ and 4-PDO selectivity from 63 to $100 \%$ as well as yield from 35 to $100 \%$ is notably observed when the reaction temperature is increased from 2 to $6 \mathrm{~h}$. The catalytic activity (SE conversion, selectivity and yield of 4-PDO) remains constant when the reaction temperature is further increased from 6 to $7 \mathrm{~h}$. The selectivity of the byproducts, viz. 3-PPCHO and cinnamyl alcohol, increases when the time is reduced from 5 to $2 \mathrm{~h}$. Moreover, 3-PPCHO selectivity is higher than cinnamyl alcohol selectivity. The catalytic reports show that the generation of FCHO from PFCHO is less at lower reaction times $(<5 \mathrm{~h})$ and therefore, the formation of byproducts is predominantly observed at a lower time. The development of the byproducts reduces with a higher reaction time, which is more favorable for the development of 4-PDO selectivity, which is in agreement with that reported elsewhere. ${ }^{9,11}$ This information demonstrates that W-ZnAlMCM-41(75) is more active than the SZ catalyst at a lower reaction time. ${ }^{11}$ From all the above catalytic results, it is worth mentioning that the best time to obtain excellent selectivity with $100 \%$ SE conversion is $6 \mathrm{~h}$.

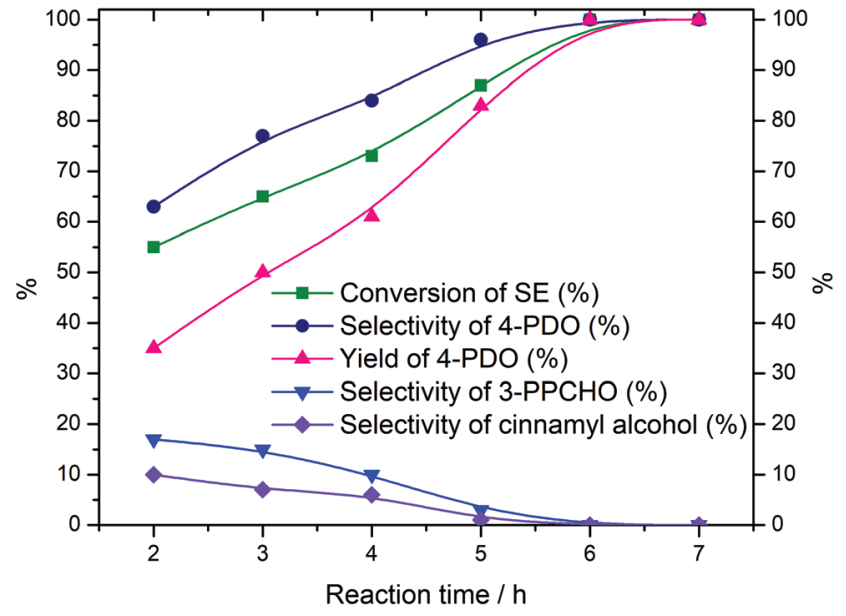

Fig. 2 Effect of reaction time over W-ZnAlMCM-41(75). Reaction conditions: $100 \mathrm{mg}$ of catalyst, $10 \mathrm{mmol}$ of SE, $20 \mathrm{mmol}$ of PFCHO, $5 \mathrm{ml}$ of 1,2-dichloroethane, temperature $=353 \mathrm{~K}, \mathrm{SE}$ to $\mathrm{PFCHO}$ mole ratio $=$ $1: 2$.

W-ZnAlMCM-41(75) was used in the Prins cyclization reaction with various molar ratios of SE to PFCHO under the reaction conditions specified in Fig. 3 to find an appropriate molar ratio of SE to PFCHO. When the concentration of PFCHO is increased only in the molar ratios from $1: 1$ to $1: 4$, the SE conversion with selectivity as well as yield of 4-PDO reaches $100 \%$ at a molar ratio of $1: 2$, slightly decreases at a molar ratio of $1: 3$ and is lower at a molar ratio of $1: 4$ compared to a molar ratio of $1: 3$. Moreover, the conversion and selectivity as well as yield decrease in other molar ratios of $2: 1$ and $2: 5$. The formation of 3-PPCHO with $5-20 \%$ selectivity is observed at other ratios, except for a molar ratio of $1: 2$. In the literature, the SZ solid catalyst shows higher selectivity of 4-PDO at a molar ratio of $1: 3$ but in this study, W-ZnAlMCM-41 produces $100 \%$ catalytic activity in a molar ratio of $1: 2$. The above catalytic results

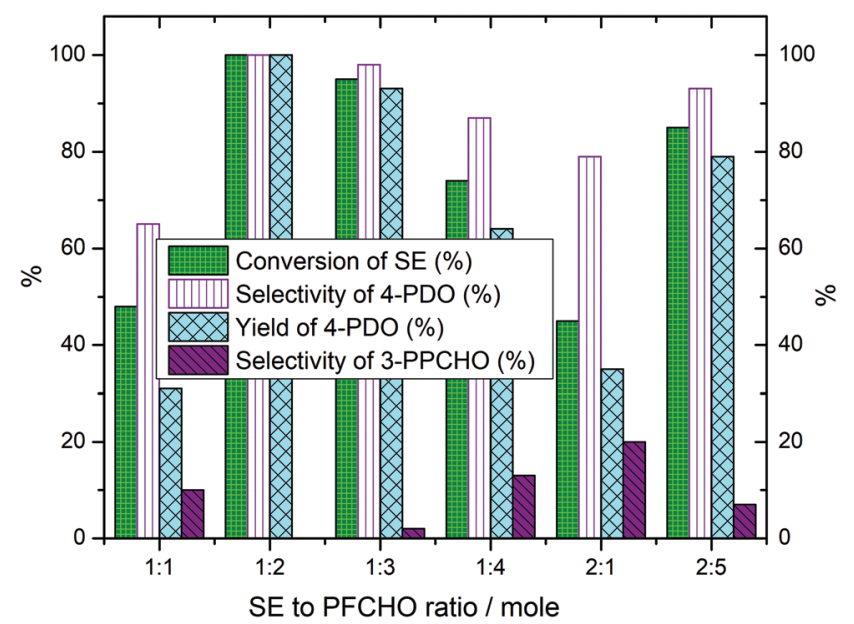

Fig. 3 Effect on the ratios of SE to PFCHO over W-ZnAIMCM-41(75). Reaction conditions: $100 \mathrm{mg}$ of catalyst, $5 \mathrm{ml}$ of 1,2-dichloroethane, temperature $=353 \mathrm{~K}$, time $=6 \mathrm{~h}$. 
show that a molar ratio of $1: 2$ is the best ratio to achieve $100 \%$ catalytic activity among the other ratios.

\subsection{Selective synthesis of 1,3-dioxanes}

Since the W-ZnAlMCM-41(75) catalyst is the best catalyst among other nanoporous catalysts for the Prins cyclization of SE, it was used in the Prins cyclization of different olefins with PFCHO. In this catalytic system, the ten SE derivatives and three aliphatic linear olefins were used as olefin sources and were allowed to react smoothly with PFCHO under the reaction conditions specified in Tables 3 and 4 to obtain the corresponding 1,3-dioxanes in excellent yields with high conversions of olefins. In all cases, the Prins cyclization of olefins with PFCHO was systemically performed with different reaction temperatures and times to obtain higher selectivity of 1,3dioxanes.

The Prins cyclization of 4-methylstyrene with PFCHO using W-ZnAlMCM-41(75) was carried out with different temperatures and times (entry 1). In this reaction, the formation of 4( $p$-tolyl)-1,3-dioxane with 99\% yield is observed at $333 \mathrm{~K}$ with $4 \mathrm{~h}$. The selectivity of 4 -( $p$-tolyl)-1,3-dioxane with the conversion of 4 -methylstyrene $(100 \%)$ is higher at $333 \mathrm{~K}$ for $4 \mathrm{~h}$ than that at other temperatures and times. An almost identical catalytic activity is observed for the Prins cyclization performed using 4-tert-butylstyrene and 4-methoxystyrene to give the 1,3dioxanes shown in Table 3 (entries 2 and 4). However, 100\% 1,3-dioxane selectivity is achieved at $333 \mathrm{~K}$ for $3 \mathrm{~h}$ because at a low reaction time the bulk electron donating group ( $t$-butyl) substituted at the $p$-position of SE reacts faster with PFCHO compared to other electron donating groups substituted at the $p$-position of SEs $\left(-\mathrm{CH}_{3}\right.$ and $\left.-\mathrm{OCH}_{3}\right)$, including SE. ${ }^{26}$ From the above catalytic results, it is clearly established that the electron donating groups $\left(-\mathrm{CH}_{3}\right.$ and $\left.-\mathrm{OCH}_{3}\right)$ substituted at the $p$-position of SE enhance the smooth reaction with PFCHO at a lower reaction temperature and time. Because the electron-donating groups substituted at the $p$-position of $\mathrm{SE}$ have negative Hammett constants $\left[\sigma_{p}(\mathrm{Me})=-0.14\right.$ and $\left.\sigma_{p}(\mathrm{OMe})=-0.28\right]$ and enable the conduction of the reactions at lower temperature as mentioned elsewhere. ${ }^{27}$ The electron withdrawing group $(-\mathrm{Cl})$ substituted at the $p$-position of SE has less reactivity with PFCHO at a lower reaction temperature and time because it has positive Hammett constant $\left[\sigma_{p}(\mathrm{Cl})=0.22\right]$, as reported in the literature. ${ }^{27}$ Therefore, in order to increase the catalytic activity of this reaction, a high reaction temperature and time are highly required. For example, 4-chlorostyrene with PFCHO gives the corresponding 1,3-dioxane in $83 \%$ yield with $95 \%$ conversion of 4-chlorostyrene at $353 \mathrm{~K}$ for $6 \mathrm{~h}$ (entry 3 ).

The electron donating group $\left(-\mathrm{CH}_{3}\right)$ substituted at the $\alpha$-position of the double bond in SE ( $\alpha$-methylstyrene) increases the reactivity and has an outward promoting effect to get the corresponding 1,3-dioxane with 98\% selectivity (entry $5)$. Furthermore, the electron withdrawing $(-\mathrm{Cl})$ and donating $\left(-\mathrm{CH}_{3}\right)$ groups substituted correspondingly at the $p$-position of the ring and the $\alpha$-position of the double bond in SE ( $p$-chloro$\alpha$-methylstyrene) boost similar reactivity and catalytic activity of $\alpha$-methylstyrene (entry 6). Moreover, the methyl group sub- stituted at the $\alpha$-position of the double bond in SE $(\alpha$-methylstyrene) produces higher reactivity compared to that substituted at the $\beta$-position ( $\beta$-methylstyrene) due to the inductive effect of the methyl group. Thus, the high reaction time needs to complete the Prins cyclization of $\beta$-methylstyrene with PFCHO in order to get the corresponding 1,3-dioxane in $74 \%$ yield with $83 \%$ conversion (entry 7 ). For further understanding, the Prins cyclization of $\beta, \beta^{\prime}$-dimethylstyrene with $\mathrm{PFCHO}$ proceeds sluggishly at a higher reaction temperature and time and produces the corresponding 1,3dioxane with $69 \%$ yield (entry 8 ). The catalytic results show that the two-methyl groups at the $\beta$-position of the double bond cause higher steric hindrance than one-methyl group. Indene has less reactivity at a lower reaction time and produces the corresponding ketone with 96\% yield at a high reaction time $(12 \mathrm{~h})$ at $353 \mathrm{~K}$ (entry 9). Naphthyl olefin, $\beta$-vinylnaphthalene, gives an inferior yield of the corresponding 1,3-dioxane when it is allowed to react with PFCHO at a lower reaction temperature and time (entry 10), as shown in Table 4 . However, this catalytic system produces better yield at a high reaction temperature and time (363 K and $12 \mathrm{~h}$ ) compared to other catalytic systems, in which different catalysts are used as reported in the literature. ${ }^{28,29}$ As reported in the literature, ${ }^{9,11}$ the long chain aliphatic linear olefins are considered to be inactive in many catalytic systems (Table 4, entries 11-13) but react well in this catalytic system with W-ZnAlMCM-41(75) under the reaction conditions given in Table 4 . The olefins increase the reactivity at a high reaction time and temperature to get the corresponding 1,3-dioxanes in yields of $81-93 \%$ with $85-95 \%$ olefin conversion. The catalytic activity almost remains constant when FN is used as an FCHO source in the Prins cyclization of different olefins under the reaction conditions specified in Table 4 . The same catalytic effects are shown in the $4^{\text {th }}$ run of recycling. Overall, in the Prins cyclization of various olefins, W-ZnAlMCM-41(75) has superior catalytic activity compared to other catalysts. ${ }^{7,11}$

\subsection{Mechanism of Prins cyclization of SE with PFCHO}

Scheme 1 shows a plausible mechanism of the Prins cyclization of SE with PFCHO. Initially, monomeric FCHO formed by the decomposition of PFCHO reacted with ZnAlMCM-41 at an appropriate reaction temperature of $353 \mathrm{~K}$ due to an interaction of PFCHO with the acid sites of the surface of the catalyst. An appropriate reaction temperature is confirmed by the studies of different temperatures, as shown in Fig. 1. The acid sites of ZnAlMCM-41 can be found by the studies of FTIR and TPD-pyridine studies and their results can be described in Table $2 \mathrm{~S}$ and Fig. $4 \mathrm{~S} . \dagger$ According to the mechanism of the Prins cyclization reaction using solid acid catalysts reported elsewhere, ${ }^{2,8,11}$ ZnAlMCM-41 leads to the protonation of the $\mathrm{C}=\mathrm{O}$ bond in $\mathrm{FCHO}$ and produces a carbocation $\left(\mathrm{HC}^{+}(\mathrm{H}) \mathrm{OH}\right)$ on the surface of the catalyst. Then the cation formed on the surface of the catalyst strikes the double bond of SE to afford a 3-phenylpropanol-like cation as an intermediate. The carbocation intermediate undergoes cycloaddition of the second FCHO molecule at $353 \mathrm{~K}$ for $6 \mathrm{~h}$ to give the desired product, 
Table 4 Prins cyclization of different olefins with PFCHO and FN over W-ZnAIMCM-41(75) and recyclable ZnAIMCM-41(75) ${ }^{a}$

\begin{tabular}{|c|c|c|c|c|c|c|c|}
\hline S. No. & $\begin{array}{l}\text { Different } \\
\text { olefins }\end{array}$ & Product & $T(\mathrm{~K})$ & Time (h) & $\begin{array}{l}\text { Conversion of } \\
\text { olefins (\%) }\end{array}$ & $\begin{array}{l}\text { Selectivity of } \\
\text { 1,3-dioxanes (\%) }\end{array}$ & $\begin{array}{l}\text { Yield of } \\
\text { 1,3-dioxanes (\%) }\end{array}$ \\
\hline \multirow[t]{6}{*}{1} & & & 313 & 4 & 51 & 69 & 35 \\
\hline & & & 313 & 6 & 65 & 72 & 47 \\
\hline & & & 333 & 4 & 99 & 100 & 99 \\
\hline & & 3-dioxane & 353 & 4 & 91 & 95 & 86 \\
\hline & & & $333^{b}$ & 4 & 98 & 100 & 98 \\
\hline & & & $333^{c}$ & 4 & 99 & 100 & 99 \\
\hline \multirow[t]{6}{*}{2} & & & 313 & 3 & 47 & 56 & 26 \\
\hline & & & 313 & 6 & 55 & 65 & 36 \\
\hline & & & 333 & 3 & 98 & 100 & 98 \\
\hline & & & 353 & 3 & 93 & 97 & 90 \\
\hline & & 4-(4-(tert-butyl)phenyl)-1,3-dioxane & $333^{b}$ & 3 & 99 & 100 & 99 \\
\hline & & & $333^{c}$ & 3 & 99 & 100 & 99 \\
\hline \multirow[t]{6}{*}{3} & & & 323 & 3 & 35 & 41 & 14 \\
\hline & & & 323 & 6 & 42 & 53 & 22 \\
\hline & & & 353 & 3 & 39 & 41 & 16 \\
\hline & & 3-dioxane & 353 & 6 & 87 & 95 & 83 \\
\hline & & & $353^{b}$ & 6 & 85 & 90 & 76 \\
\hline & & & $353^{c}$ & 6 & 87 & 95 & 83 \\
\hline \multirow[t]{6}{*}{4} & & & 313 & 4 & 56 & 60 & 34 \\
\hline & & & 313 & 6 & 77 & 86 & 66 \\
\hline & & & 333 & 4 & 97 & 100 & 97 \\
\hline & & $4-(4$ & 353 & 4 & 91 & 96 & 87 \\
\hline & & 4-(-4-me & $333^{b}$ & 4 & 98 & 100 & 98 \\
\hline & & & $333^{c}$ & 4 & 97 & 100 & 97 \\
\hline \multirow[t]{6}{*}{5} & & & 323 & 3 & 31 & 43 & 13 \\
\hline & & & 323 & 6 & 45 & 57 & 26 \\
\hline & & & 353 & 3 & 35 & 41 & 14 \\
\hline & & .3-dioxane & 353 & 6 & 93 & 98 & 91 \\
\hline & & & $353^{b}$ & 6 & 94 & 98 & 92 \\
\hline & & & $353^{c}$ & 6 & 93 & 99 & 92 \\
\hline \multirow[t]{6}{*}{6} & & & 323 & 3 & 35 & 41 & 14 \\
\hline & & & 323 & 6 & 51 & 58 & 29 \\
\hline & & & 353 & 3 & 43 & 61 & 26 \\
\hline & & & 353 & 6 & 95 & 100 & 95 \\
\hline & & 4-(4-chlorophenyl)-4-methyl-1,3-dioxane & $353^{b}$ & 6 & 90 & 100 & 90 \\
\hline & & & $353^{c}$ & 6 & 96 & 100 & 96 \\
\hline \multirow[t]{6}{*}{7} & & & 323 & 6 & 48 & 51 & 24 \\
\hline & & & 323 & 15 & 63 & 67 & 42 \\
\hline & & & 353 & 6 & 55 & 77 & 42 \\
\hline & & 5-Methyl-4-phenyl-1,3-dioxane & 353 & 15 & 83 & 89 & 74 \\
\hline & & & $353^{b}$ & 15 & 71 & 85 & 60 \\
\hline & & & $353^{c}$ & 15 & 84 & 89 & 75 \\
\hline \multirow[t]{6}{*}{8} & & & 323 & 6 & 30 & 37 & 11 \\
\hline & & & 323 & 15 & 42 & 51 & 21 \\
\hline & & & 363 & 6 & 38 & 47 & 18 \\
\hline & & enyl-1,3-dioxane & 363 & 15 & 71 & 98 & 69 \\
\hline & & & $363^{b}$ & 15 & 68 & 91 & 62 \\
\hline & & & $363^{c}$ & 15 & 71 & 98 & 69 \\
\hline \multirow[t]{6}{*}{9} & & & 323 & 6 & 31 & 42 & 13 \\
\hline & & & 323 & 12 & 48 & 76 & 36 \\
\hline & & & 353 & 6 & 55 & 83 & 46 \\
\hline & & 4,4a,9,9a-tetrahydroindeno[[2,1-d][1,3]dioxine & 353 & 12 & 97 & 99 & 96 \\
\hline & & & $353^{b}$ & 12 & 99 & 98 & 97 \\
\hline & & & $353^{c}$ & 12 & 98 & 99 & 97 \\
\hline \multirow[t]{6}{*}{10} & & & 323 & 6 & 34 & 41 & 14 \\
\hline & & & 323 & 12 & 45 & 51 & 23 \\
\hline & & & 363 & 6 & 39 & 44 & 17 \\
\hline & & 4-(2-Naphthyl)-1,3-dioxane: & 363 & 12 & 71 & 95 & 67 \\
\hline & & & $363^{b}$ & 12 & 68 & 91 & 62 \\
\hline & & & $363^{c}$ & 12 & 71 & 96 & 68 \\
\hline \multirow[t]{6}{*}{11} & & & 323 & 6 & 25 & 35 & 9 \\
\hline & & & 323 & 12 & 52 & 67 & 35 \\
\hline & & 4-hexyl-1,3-dioxane & 363 & 6 & 37 & 48 & 18 \\
\hline & & & 363 & 12 & 95 & 98 & 93 \\
\hline & & & $363^{b}$ & 12 & 89 & 95 & 84 \\
\hline & & & $363^{c}$ & 12 & 96 & 98 & 94 \\
\hline
\end{tabular}


Table 4 (Contd.)

\begin{tabular}{|c|c|c|c|c|c|c|c|}
\hline S. No. & $\begin{array}{l}\text { Different } \\
\text { olefins }\end{array}$ & Product & $T(\mathrm{~K})$ & Time (h) & $\begin{array}{l}\text { Conversion of } \\
\text { olefins (\%) }\end{array}$ & $\begin{array}{l}\text { Selectivity of } \\
\text { 1,3-dioxanes (\%) }\end{array}$ & $\begin{array}{l}\text { Yield of } \\
\text { 1,3-dioxanes (\%) }\end{array}$ \\
\hline \multirow[t]{6}{*}{12} & & & 323 & 6 & 20 & 35 & 7 \\
\hline & & & 323 & 12 & 42 & 59 & 25 \\
\hline & & 4-heptyl-1,3-dioxane & 363 & 6 & 29 & 37 & 11 \\
\hline & & & 363 & 12 & 93 & 98 & 91 \\
\hline & & & $363^{b}$ & 12 & 88 & 95 & 84 \\
\hline & & & $363^{c}$ & 12 & 94 & 98 & 92 \\
\hline \multirow[t]{6}{*}{13} & & & 323 & 6 & 15 & 29 & 4 \\
\hline & & & 323 & 12 & 31 & 45 & 14 \\
\hline & & 4-nonyl-1,3-dioxane & 363 & 6 & 27 & 37 & 10 \\
\hline & & & 363 & 12 & 85 & 95 & 81 \\
\hline & & & $363^{b}$ & 12 & 78 & 90 & 70 \\
\hline & & & $363^{c}$ & 12 & 86 & 96 & 82 \\
\hline
\end{tabular}

${ }^{a}$ Reaction conditions: $100 \mathrm{mg}$ of W-ZnALMCM-41(75)/recyclable ZnAlMCM-41(75), $10 \mathrm{mmol}$ of olefin, $20 \mathrm{mmol}$ of PFCHO, $5 \mathrm{ml}$ of 1,2-dichloroethane. ${ }^{b} 30 \mathrm{mmol}$ of FN instead of $20 \mathrm{mmol}$ of PFCHO. ${ }^{c}$ The catalytic results received in the $4^{\text {th }}$ run.

4-PDO with the regeneration of the ZnAlMCM-41 catalyst. When the formation of FCHO from the PFCHO is less at the lower reaction times and unsuitable ratios of SE to PFCHO, as shown in Fig. 2 and 3, the intermediate carbocation creates cinnamyl alcohol and 3-PPCHO, as the byproducts, by releasing a proton and transformation of the proton, respectively. A similar reaction mechanism can be followed for the Prins cyclization of different olefins with PFCHO/FN. The hydrophobicity, hydrothermal stability and active sites of ZnAlMCM-41(75) are key factors in the production of 1,3-dioxanes with excellent yields.

\section{Conclusions}

Many nanoporous catalysts, including mesoporous ZnAlMCM-41, have been successfully used in the Prins cyclization of SE with PFCHO in the production of 4-PDO. Recyclability studies have been performed to find the stability of the catalyst. A chemical treatment method is successfully used to remove trace amounts of non-framework zinc and aluminum ions on the surface of ZnAlMCM-41(75), and then the treated catalyst is called W-ZnAlMCM-41, which is successfully used in this reaction to further evaluate the catalytic stability. The catalytic results show that both the catalysts, viz. W-ZnAMCM-41(75) and recyclable ZnAlMCM-41(75), have almost similar catalytic activity, which remains constant after the $4^{\text {th }}$ run and confirms that the loss of active sites on the surface of the catalyst could not be observed. More than thirteen solvents have been tested to find the best solvent. Even though 1,2-dicloroethane is the best solvent among the other solvents for the highly selective synthesis of 4-PDO, it is volatile, toxic and not considered as a green solvent. Actually, FN is a good source of FCHO and has been successfully used instead of PFCHO with water as a solvent to get a higher yield of 4-PDO. This is highly considered as a very ecofriendly and heterogeneous catalytic process. Effects of various reaction parameters are systematically investigated to find the best optimal conditions. W-ZnAlMCM-41(75) is successfully used in the Prins cyclization of different olefins with $\mathrm{PFCHO/FN}$ as the FCHO sources. The above catalytic studies prove that the W-ZnAlMCM-41(75) catalyst has higher catalytic activity than other nanoporous catalysts, produces $100 \%$ yield of 4 -PDO and $67-100 \%$ yield of other 1,3-dioxanes, and is a highly active and very ecofriendly heterogeneous catalyst for the selective synthesis of 1,3-dioxanes.

\section{Conflicts of interest}

There are no conflicts to declare.

\section{Acknowledgements}

The authors extend their appreciation to the Deanship of Scientific Research at King Khalid University for funding this work through a General Research Project under grant number G.R.P. $317 / 1442$.

\section{References}

1 H. J. Prins, Chem. Weekbl., 1919, 16, 1072.

2 (a) E. Arundale and L. A. Mikeska, Chem. Rev., 1952, 51, 505-555; (b) M. P. Isidro and Y. Miguel, Curr. Org. Chem., 2007, 11, 925-957; (c) B. B. Snider, in Comprehensive Organic Synthesis, ed. B. M. Trost and I. Fleming, Pergamon, Oxford, 1991, pp. 527-561.

3 G. A. Olah and Á. Molnár, Hydrocarbon chemistry, John Wiley \& Sons, 2003.

4 J. March, Advanced organic chemistry: reactions, mechanisms, and structure, John Wiley \& Sons, 1992.

5 B. Snider, Comprehensive organic synthesis, Pergamon Press, Oxford, 1991, vol. 2, p. 527.

6 A. P. Amrute, S. Sahoo, A. Bordoloi, Y. K. Hwang, J.-S. Hwang and S. B. Halligudi, Catal. Commun., 2009, 10, 1404-1409. 
7 S. Sreevardhan Reddy, B. David Raju, V. Siva Kumar, A. H. Padmasri, S. Narayanan and K. S. Rama Rao, Catal. Commun., 2007, 8, 261-266.

8 M. A. Aramendía, V. Borau, C. Jiménez, J. M. Marinas, F. J. Romero and F. J. Urbano, Catal. Lett., 2001, 73, 203206.

9 G. Li, Y. Gu, Y. Ding, H. Zhang, J. Wang, Q. Gao, L. Yan and J. Suo, J. Mol. Catal. A: Chem., 2004, 218, 147-152.

10 T. Jun-ichi, H. Keiji, Y. Takayoshi and U. Sakae, Bull. Chem. Soc. Jpn., 1996, 69, 2361-2368.

11 V. S. Marakatti, G. V. Shanbhag and A. B. Halgeri, Appl. Catal., A, 2013, 451, 71-78.

12 (a) W. Zhang, Y. Leng, P. Zhao, J. Wang, D. Zhu and J. Huang, Green Chem., 2011, 13, 832-834; (b) M. Nandi, J. Mondal, K. Sarkar, Y. Yamauchi and A. Bhaumik, Chem. Commun., 2011, 47, 6677-6679; (c) S. K. Kundu, J. Mondal and A. Bhaumik, Dalton Trans., 2013, 42, 10515-10524; (d) M. Pramanik and A. Bhaumik, ACS Appl. Mater. Interfaces, 2014, 6, 933-941; (e) C. Subrahmanyam, B. Louis, F. Rainone, B. Viswanathan, A. Renken and T. K. Varadarajan, Catal. Commun., 2002, 3, 45-50; (f) K. V. A. Kumar, S. R. Amanchi, B. Sreedhar, P. Ghosal and C. Subrahmanyam, RSC Adv., 2017, 7, 43030-43039; (g) M. Selvaraj, D. W. Park, I. Kim, S. Kawi and C. S. Ha, Dalton Trans., 2012, 41, 14204-14210.

13 J. M. Watson and A. Chepregi, U.S. Patent 4185019, 1980.

14 M. Selvaraj, B. R. Min, Y. G. Shul and T. G. Lee, Microporous Mesoporous Mater., 2004, 74, 143-155.
15 M. Selvaraj and S. Kawi, J. Mol. Catal. A: Chem., 2006, 246, 218-222.

16 (a) M. Selvaraj, Ph.D. thesis, Anna University, Chennai, 2002; (b) M. Selvaraj and S. Kawi, J. Mol. Catal. A: Chem., 2007, 265, 250-257; (c) M. Selvaraj and M. A. Assiri, Dalton Trans., 2019, 48, 12986-12995; (d) M. Selvaraj and M. A. Assiri, Ind. Eng. Chem. Res., 2020, 59, 14703-14709.

17 M. Selvaraj, S. W. Song and S. Kawi, Microporous Mesoporous Mater., 2008, 110, 472-479.

18 Y. Du and F. Tian, Catal. Commun., 2007, 8, 2012-2016.

19 E. A. Gunnewegh, S. S. Gopie and H. van Bekkum, J. Mol. Catal. A: Chem., 1996, 106, 151-158.

20 J. Medina-Valtierra, M. A. Sánchez, J. A. Montoya, J. Navarrete and J. A. de los Reyes, Appl. Catal., A, 1997, 158, L1-L6.

21 A. Corma, Chem. Rev., 1997, 97, 2373-2420.

22 V. Gutmann, Coord. Chem. Rev., 1976, 18, 225-255.

23 J. Deutsch, H. A. Prescott, D. Müller, E. Kemnitz and H. Lieske, J. Catal., 2005, 231, 269-278.

24 Y.-M. Chung and H.-K. Rhee, J. Mol. Catal. A: Chem., 2001, 175, 249-257.

25 I. Persson, Pure Appl. Chem., 1986, 58, 1153-1161.

26 J. Zhang, L. Hua, F. Li, X. Wu, S. Tian and J. Yang, Synth. Commun., 2012, 42, 1234-1242.

27 T. Bach and J. Löbel, Synthesis, 2002, 2521-2526.

28 G. Yanlong, O. Chikako and K. Shu, Chem. Lett., 2006, 35, 1176-1177.

29 J. S. Yadav, B. V. S. Reddy and G. Bhaishya, Green Chem., 2003, 5, 264-266. 